OUTP-97-15P

hep-th/9703209

March 28, 1997

\title{
Matrix Strings in Two-dimensional Yang-Mills Theory
}

\author{
IAN I. Kogan and Richard J. Szabof \\ Department of Physics - Theoretical Physics \\ University of Oxford \\ 1 Keble Road, Oxford OX1 3NP, U.K.
}

\begin{abstract}
We describe the structure of string vacuum states in the supersymmetric matrix model for $\mathrm{M}$ theory compactified on a circle in the large- $N$ limit. We show that the theory admits topological instanton field configurations which at short-distance scales reduce to ordinary Yang-Mills instantons that interpolate between degenerate vacua of the theory. We show that there exists further classes of hadronic strings associated with the D-string super-fields. We discuss the relationships between these non-perturbative string states and rigid QCD strings, critical strings, and membrane states.
\end{abstract}

${ }^{*}$ Work supported in part by the Natural Sciences and Engineering Research Council of Canada. 
'M theory' is the non-perturbative theory that is a quantum extension of 11-dimensional supergravity [1]. Via compactification on a circle $S^{1}$, it is equivalent to 10-dimensional type-IIA superstring theory. M theory in this way subsumes all five consistent superstring theories in 10 dimensions and contains 11-dimensional supergravity as its low-energy limit. It has been suggested recently that the full non-perturbative dynamics of this theory lies in the content of an appropriate large- $N$ limit of a supersymmetric matrix model [2]. This $N \times N$ matrix quantum mechanics is obtained as the dimensional reduction of supersymmetric $U(N)$ Yang-Mills gauge theory in 10 dimensions [3] and it describes the low-energy collective dynamics of $N$ parallel D0-branes in the weak-coupling limit of type-IIA superstring theory [4, 5]. As each block $N_{i} \times N_{i}$ sub-matrix, with $\sum_{i} N_{i}=N$, describes a non-relativistic super-membrane (regarded as a collective excitation of D0-branes) [6], the $N \rightarrow \infty$ limit of the matrix model describes a multi-membrane theory and thus naturally incorporates the quantum field theoretical Fock space for the membranes of supergravity. The permutation symmetry group $S_{N}$ of block diagonal matrices is naturally realized as the Weyl group of the gauge group $U(N)$, and the non-commutativity of the matrix coordinate fields at small distance scales gives a realization of the idea that at distances smaller than the Planck length the conventional notion of a spacetime geometry breaks down. Since the longitudinal momentum in the 11-th direction is $p_{11}=N / R_{11}$, with $R_{11}$ the compactification radius, the large- $N$ limit also effectively accomplishes a decompactification of the extra 11-th direction and should describe the short-distance space-time structure of $\mathrm{M}$ theory in the infinite momentum frame. This conjecture has thus far survived a number of consistency checks [7] leading to a "third quantization" of string theory.

In this Letter we will study some features of the spectrum of states of the supersymmetric matrix model for $\mathrm{M}$ theory when one of the nine transverse directions is compactified on a circle $S^{1}$. In this case, the matrix quantum mechanics becomes a two-dimensional matrix field theory coupled to two-dimensional Yang-Mills theory which is also obtained as the dimensional reduction of 10-dimensional supersymmetric Yang-Mills theory down to two-dimensions, and it describes the non-relativistic dynamics of D-strings [2], [8]- [10]. The non-perturbative formulation of string theory as a two-dimensional supersymmetric Yang-Mills theory was first proposed in [11. This viewpoint was elaborated on in [12] where it was discussed how to identify the particle spectrum of string theory with the states that can be made up from infinitely many D-particles. In the following we shall study the particle spectrum of the D-string field theory by considering the effective twodimensional Yang-Mills theory that is obtained. We shall see that the two-dimensional gauge theory that we study in this way is an important model for deducing the spectrum of string vacuum states, and also for a non-perturbative quantum gauge theory framework for string theory. Using the $S O(8)$ triality property of the theory 13 we show that in the large- $N$ limit the gauged action for the D-string super-fields can be diagonalized by an explicit Nicolai mapping [14, 15]. From this we identify some of the non-perturbative 
degrees of freedom in the large- $N$ limit that describe the string vacuum states as topological instanton fields from the string world-sheet into the eight-dimensional target space. These instantons interpolate between ordinary Yang-Mills instantons on the world-sheet at short-distance scales, which connect the usual $\theta$-vacua of two-dimensional adjoint quantum chromodynamics (QCD), and the D-string configurations at large-distance scales which describe the free string limit of the theory. We emphasize the potential relevance of the instanton sum that we find to the construction of non-perturbative membrane states. We also demonstrate the appearence of certain hadronic strings constructed from the D-string super-fields of the theory, and we discuss the properties of these string states in conjuction with rigid QCD strings and critical strings. We also discuss the potential interplay between the instanton moduli space that we find and the conventional moduli spaces that arise in two-dimensional Yang-Mills theory [15.

The D-string field theory is the two-dimensional $\mathcal{N}=8$ supersymmetric $U(N)$ YangMills theory with action

$$
\begin{aligned}
S[A ; X, \psi]=\frac{R^{2}}{2 \pi \alpha^{\prime}} \int d \tau & \oint d \sigma \operatorname{tr}\left(\frac{1}{2} \sum_{i=1}^{8}\left(\nabla_{A, \mu} X^{i}\right)^{\dagger}\left(\nabla_{A}^{\mu} X^{i}\right)+\psi^{T} \nabla_{A} \psi+g^{2} F_{A, \mu \nu} F_{A}^{\mu \nu}\right. \\
& \left.-\frac{1}{2 g^{2}} \sum_{i<j}\left[X^{i}, X^{j}\right]^{2}+\frac{1}{g} \sum_{i=1}^{8} \psi^{T} \gamma_{i}\left[X^{i}, \psi\right]\right)
\end{aligned}
$$

where $g$ is the string coupling constant and $\alpha^{\prime}$ is the string tension. The bosonic fields $X^{i}(\tau, \sigma), i=1, \ldots, 8$, are $N \times N$ Hermitian matrices in the adjoint representation of the local gauge symmetry group $U(N)$ which transform in the vector $\boldsymbol{8}_{v}$ representation of the global $S O(8)$ R-symmetry group of rotations in the transverse space. They describe the collective coordinates in $\mathbb{R}^{8}$ of the $N$ D-strings. Their superpartners are the $N \times N$ fermionic matrices $\psi(\tau, \sigma)=\left(\begin{array}{c}\psi_{\mathrm{L}}^{\alpha}(\tau+\sigma) \\ \psi_{\mathrm{R}}^{\dot{\alpha}}(\tau-\sigma)\end{array}\right), \alpha, \dot{\alpha}=1, \ldots, 8$, where $\psi_{\mathrm{L}}^{\alpha}(\tau+\sigma)$ and $\psi_{\mathrm{R}}^{\dot{\alpha}}(\tau-\sigma)$ are Majorana-Weyl spinor fields in the adjoint representation of $U(N)$ which transform, respectively, in the spinor $\boldsymbol{8}_{s}$ and conjugated spinor $\boldsymbol{8}_{c}$ representation of $S O(8)$. The trace in (11) is over the $U(N)$ indices. The action $S$ also contains a residual, non-dynamical twodimensional $U(N)$ gauge field $A_{\mu}(\tau, \sigma), \mu=0,1$, and $F_{A}=d A+[A, A] / 2$ is its curvature. The world-sheet is the cylinder $\left(x^{0}, x^{1}\right) \equiv(\tau, \sigma) \in \mathbb{R}^{1} \times S^{1}$ with $R$ the radius of the circle $S^{1}$, so that $\sigma \in[0,2 \pi R)$. For a global $\mathcal{N}=8$ world-sheet supersymmetry, we need to take the fermion fields to lie in the Ramond sector of the world-sheet theory, i.e. $\psi^{\alpha}\left(\mathrm{e}^{-2 \pi i R} z\right)=\psi^{\alpha}(z)$ and $\psi^{\dot{\alpha}}\left(\mathrm{e}^{2 \pi i R} \bar{z}\right)=\psi^{\dot{\alpha}}(\bar{z})$ where $z=\mathrm{e}^{-i(\tau+\sigma)}$ and $\bar{z}=\mathrm{e}^{-i(\tau-\sigma)}$. The gamma-matrices $\gamma^{i}$ generate the spin(8) Clifford algebra in a Majorana-Weyl basis. They can be decomposed with respect to the reducible $\boldsymbol{8}_{s} \oplus \boldsymbol{8}_{c}$ representation of $S O(8)$ as

$$
\gamma^{i}=\left(\begin{array}{cc}
0 & \gamma_{\alpha \dot{\alpha}}^{i} \\
\gamma_{\dot{\alpha} \alpha}^{i} & 0
\end{array}\right)
$$

where $\gamma_{\dot{\alpha} \alpha}^{i}=\left(\gamma^{i}\right)_{\alpha \dot{\alpha}}^{T}$ are real-valued, and for $i=1, \ldots, 7$ they are anti-symmetric while $\gamma_{\dot{\alpha} \alpha}^{8}$ is symmetric. The gauge-covariant derivative in (11) is $\nabla_{A}=d-i[A, \cdot]$, and 
$\nabla_{A}=\nabla_{A}^{\mu} \Gamma_{\mu}$ where the world-sheet gamma-matrices are $\Gamma^{0}=\left(\gamma^{0}\right)^{2}=\left(\begin{array}{cc}\mathbf{1}_{8} & 0 \\ 0 & \mathbf{1}_{8}\end{array}\right)$ and $\Gamma^{1}=\gamma^{9}=\left(\begin{array}{cc}\mathbf{1}_{8} & 0 \\ 0 & -\mathbf{1}_{8}\end{array}\right)$ with $\mathbf{1}_{8}$ the $8 \times 8$ identity matrix.

The action (11) describes a non-trivial matrix field theory coupled to two-dimensional Yang-Mills theory. The temporal component $A_{0}$ of the gauge field is non-dynamical but acts as a Lagrange multiplier enforcing the invariance of the theory under local gauge transformations. Varying $S$ with respect to it gives the generator $\mathcal{G}$ of these transformations in the Weyl gauge as

$$
\mathcal{G}=4 g^{2} \nabla_{A}^{\sigma}\left(\partial_{\tau} A_{1}\right)-2\left[X_{i}, \partial_{\tau} X_{i}\right]+\left[\psi^{T}, \psi\right]
$$

and the constraint $\mathcal{G} \sim 0$ enforces local gauge invariance of the quantum field theory. It is instructive to consider the model (ID) as a bosonic matrix field theory coupled to two-dimensional adjoint QCD with colour group $U(N)$. There are then eight flavours of massless adjoint fermions in this interpretation.

Let us start by examining the confinement problem in this model. Consider first the weak-coupling limit $g^{2} \rightarrow \infty$ of the Yang-Mills theory. In that limit, the action (1) reduces to the ordinary adjoint QCD action

$$
S_{\mathrm{QCD}_{2}}[A, \psi]=\frac{R^{2}}{2 \pi \alpha^{\prime}} \int d \tau \oint d \sigma \operatorname{tr}\left(\psi^{T} \not_{A} \psi+g^{2} F_{A, \mu \nu} F_{A}^{\mu \nu}\right)
$$

plus an additional term for the interaction of the dynamical boson fields $X^{i}$ with the Yang-Mills field. In each field sector there are $N^{2}$ colour degrees of freedom. As shown in [16], the fundamental path-ordered Wilson loop

$$
W_{\mathcal{C}}[A]=\frac{\operatorname{tr}_{F}}{N} P \exp \left(i \oint_{\mathcal{C}} A_{\mu}(x) d x^{\mu}\right)
$$

in this case is screened by the massless adjoint fermions, i.e. its vacuum expectation value in the fermion-gauge sector of the theory obeys a perimeter law, rather than an area law which is the signal of quark confinement. This means that a heavy probe charge in the fundamental colour representation, whose holonomy from parallel transport along the closed contour $\mathcal{C}$ due to its coupling to $A$ is determined by (5), is screened by the dynamical quarks, in contrast to the confinement phase where one obtains a linear static potential between external quarks. The classic example of this phenomenon occurs in the massless Schwinger model [16] which is a $U(1)$ version of (4). Then the screening of test charges is due to a dynamical Higgs mechanism for the photon field which induces a non-local Schwinger mass term proportional to

$$
\mathcal{S}[A]=\frac{1}{4 g^{2}} \int d \tau \oint d \sigma \epsilon_{\mu \nu} F_{A}^{\mu \nu} \square^{-1} \epsilon_{\lambda \rho} F_{A}^{\lambda \rho}
$$

after integrating over the fermion fields. This effect occurs outside a radius $R_{s} \sim g^{2}$. However, in the weak-coupling regime, the screening radius becomes infinite, and so at 
$g^{2} \rightarrow \infty$ the matrix string theory is essentially confining (because the induced dynamical gluon mass vanishes).

The situation is somewhat different in the strong-coupling phase $g^{2} \rightarrow 0$. This regime corresponds to the free string limit and the resulting field theory (1) is quite different from the adjoint QCD which dominates the weak-coupling phase. At $g^{2} \rightarrow 0$ we have $\left[X^{i}, X^{j}\right]=0$, so that the D-string coordinates can be simultaneously diagonalized and hence taken to lie in the Cartan subalgebra of $U(N)$, i.e.

$$
X^{i}(\tau, \sigma)=\operatorname{diag}\left[x_{1}^{i}(\tau, \sigma), \ldots, x_{N}^{i}(\tau, \sigma)\right]
$$

The D-strings now have well-defined configurations described by the coordinate fields $x_{a}^{i}$ (in contrast to the generic case where the fields reside in a non-commutative spacetime geometry). There are now only $N$ colour degrees of freedom in each field sector and the model is described in terms of the Green-Schwarz light-cone coordinates $x_{a}^{i}, \psi_{a}^{\alpha}$ and $\psi_{a}^{\dot{\alpha}}$ with $a=1, \ldots, N$. The effective field theory is the $\mathcal{N}=8$ supersymmetric sigma-model in the orbifold target space $\left(\mathbb{R}^{8}\right)^{N} / S_{N}$ [12]. From the point of view of the Schwinger model, we effectively have $N$ abelian gauge copies in which all fermion and boson fields are neutral because they lie in the adjoint representation of the local gauge group. There are no massless dynamical charged degrees of freedom, and hence no screening. Furthermore, the gauge symmetry is broken as $U(N) \rightarrow U(1)^{N}$ at $g^{2} \rightarrow 0$ so that an external quark yields a topological sector with the quantum numbers of $N$ D-strings and one elementary string associated with a $U(1)$ factor [4].

Thus at both weak and strong coupling we expect external colour probe charges to be confined in the quantum field theory (西). If we now apply the analysis of [13] which suggests that there are no phase transitions in this theory as the string coupling constant $g$ is continuously varied, then we can deduce that the matrix field theory (1) is confining for all values of $g^{2}$. This leads to a picture of string states in the gauged supersymmetric matrix model (1) as QCD strings formed in a confining phase. These matrix strings are hadronic states which form $S O(8)$ flavour multiplets and $U(N)$ colour singlets. These strings are not expected to be the usual rigid strings of QCD (except possibly at weakcoupling), but more likely critical strings.

The hadronic spectrum is actually richer, because we have not yet exploited the topology of the underlying cylindrical world-sheet which has non-trivial fundamental group $\pi_{1}\left(\mathbb{R}^{1} \times S^{1}\right)=\mathbb{Z}$ that gives the two-dimensional gauge field $A$ finitely-many topological degrees of freedom. The locally gauge-invariant Wilson loops (5) are, as in the confinement discussion above, the crucial objects which identify the non-perturbative states of the theory. In particular, as discussed in [17, on a cylinder there are now actually two types of hadronic states in the spectrum, namely those associated with contractible and non-contractible loops $\mathcal{C}^{(n)} \in \pi_{1}\left(\mathbb{R}^{1} \times S^{1}\right)$ on the world-sheet in (5) (Fig. 1), where $n \in \mathbb{Z}$ is the number of times that the loop winds around the circle $S^{1}$ of the world-sheet with a particular orientation. The topologically non-trivial ones $(n \neq 0)$ are those associated 
with the gauge field configurations $A_{\mu}$ that encircle the spatial part $S^{1}$ of the world-sheet cylinder. Their spatial components admit the Hodge decomposition

$$
A_{1}(\tau, \sigma)=\frac{1}{R} \sigma+\partial_{\tau} \chi(\tau, \sigma)+\partial_{\sigma} \alpha(\tau, \sigma)
$$

In addition to these pure gluon winding modes, there are also the more complicated locally gauge invariant operators involving the adjoint boson or fermion fields $\Phi$ (Fig. 1)

$$
\begin{aligned}
W_{\mathcal{C}^{(n)}}^{\left(x_{1}, \ldots, x_{m}\right)}[A, \Phi]=\frac{\operatorname{tr}_{F}}{N} P & \Phi\left(x_{1}\right) \exp \left(i \int_{x_{1}}^{x_{2}} A_{\mu}(x) d x^{\mu}\right) \Phi\left(x_{2}\right) \cdots \\
& \times \cdots \Phi\left(x_{m}\right) \exp \left(i \int_{x_{m}}^{x_{1}} A_{\mu}(x) d x^{\mu}\right)
\end{aligned}
$$

inserted at points $x_{1}, \ldots, x_{m}$ on the non-contractible loop $\mathcal{C}^{(n)}$.

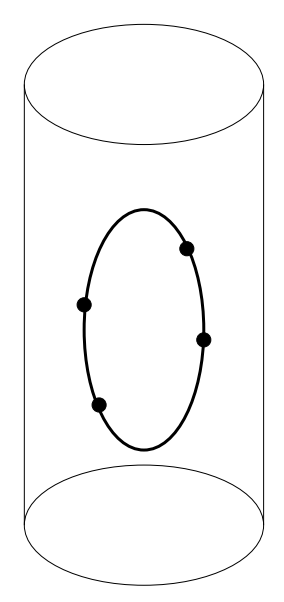

(a)

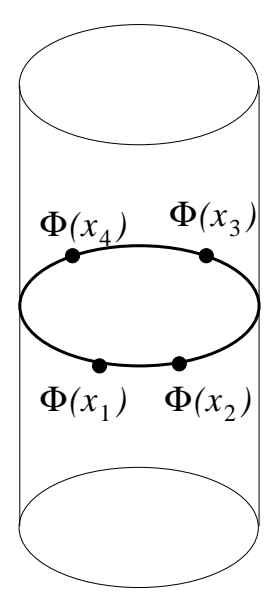

(b)

Figure 1: The two types of hadronic string states on the matrix world-sheet $\mathbb{R}^{1} \times S^{1}$. The bold lines denote the gluon strings and the solid circles depict insertions of the adjoint matter fields along the Wilson loops. The hadrons propagate between the bottom and the top of the cylinder and form either contractible loops as shown in (a) or the winding modes depicted in (b) whereby they encircle the loop of the world-sheet $n$ times. These strings define $N$ inequivalent vacua which are connected by topological instantons.

The fundamental Wilson loops (5) are in general not invariant under large gauge transformations. Under a global gauge transformation which winds $n$ times around $S^{1}$, i.e. $\sigma \rightarrow \sigma+2 \pi R n$, the fundamental Wilson loop twists

$$
W_{\mathcal{C}^{(n)}}[A] \rightarrow \mathrm{e}^{2 \pi i n / N} W_{\mathcal{C}^{(n)}}[A]
$$

by an element in the center $\mathbb{Z}_{N}$ of the group $S U(N)$. This property follows from the fact that the adjoint matter fields are decoupled from the photon field in the decomposition of the $U(N)$ gauge field $A$ with respect to the exact sequence

$$
1 \rightarrow \mathbb{Z}_{N} \rightarrow S U(N) \times U(1) \rightarrow U(N) \rightarrow \mathbf{1}
$$


and it is intimately related to the fact that adjoint QCD has exotic topological properties. The global gauge group is effectively $S U(N) / \mathbb{Z}_{N}$ which has a non-trivial fundamental group

$$
\pi_{1}\left(S U(N) / \mathbb{Z}_{N}\right)=\pi_{0}\left(\mathbb{Z}_{N}\right)=\mathbb{Z}_{N}
$$

and so there are $N$ topologically inequivalent superselection sectors in the vacuum of the quantum field theory [18]. The non-trivial topology (12) of the global gauge group yields $N$ gauge-inequivalent vacuum states $|0\rangle_{n}$ labelled by the $N$-th roots of unity [17]. In particular, we see the appearence of the usual Yang-Mills instantons, which interpolate between the inequivalent vacua, as new non-perturbative excitations in the matrix string theory (1). The explicit instanton field configurations are represented by the Wilson loop operators $W_{\mathcal{C}^{(n)}}[A]$ which are interpolating fields for the inequivalent vacua as described by their twisting property $(\sqrt{10})$.

Thus, in addition to the local hadronic strings arising from the confining properties of the theory, there are global inequivalent vacua $|0\rangle_{n}$ which are described by topological strings which form non-contractible loops $\mathcal{C}^{(n)}$ of a given winding number $0 \leq n<N$. The winding numbers label the topological class of the parallel transport operators $W_{\mathcal{C}^{(n)}}[A]$ which effectively act in the fibers of the principal $S U(N) / \mathbb{Z}_{N}$ bundle over the world-sheet and describe interpolating instanton configurations between the vacua. In addition to the spectrum of winding modes corresponding to the pure gluon non-contractible loops, there are also the mixed Wilson loop operators (9), with adjoint matter fields inserted along the non-contractible loops, which can decay into the usual hadron modes and pure gluon winding modes $W_{\mathcal{C}^{(n)}}[A]$. Note that these strings differ from the fundamental strings discussed in [10] which correspond to the photon field in the supersymmetric Yang-Mills theory, and also from the twisted long string states in [11, 12]. In the limit $N \rightarrow \infty$, the field theory has an infinite number of degrees of freedom with infinitely many inequivalent vacua $|0\rangle_{n}$. Then the vacuum sector of the D-string Fock space contains an infinity of hadron and gluon string modes with infinitely many instanton configurations. These objects can be identified as non-perturbative degrees of freedom in the membrane theory under consideration. It would be interesting to see if there is any sort of instanton condensation in the ground state upon taking the large- $N$ limit corresponding to a degeneracy in the twisting property (10) of the gluon winding modes.

We have not yet fully exploited the dynamics of the D-string embedding fields $X^{i}$ in the eight-dimensional target space (or equivalently the $\mathcal{N}=8$ supersymmetry of the matrix model (1) ). It turns out, as we now demonstrate, that there is in fact a much larger class of target space instanton field configurations which reduce, in the weak-coupling phase, to the world-sheet instantons of two-dimensional Yang-Mills theory that we described above. For this, we consider the vacuum amplitude which is given by the path integral

$$
Z(R, g ; N)=\int D A D X D \psi \mathrm{e}^{-N S[A ; X, \psi]}
$$

where it is understood that the functional integration is restricted to gauge orbits and to 
fermion field configurations with periodic boundary conditions. In the case of constant world-sheet field configurations (the ultra-local form of the theory), the integration over the constant modes $X_{0}$ and $\psi_{0}$ of the matrix degrees of freedom was explicitly carried out in 13. There it was shown that the triality property $\mathbf{8}_{v} \cong \mathbf{8}_{s} \cong \mathbf{8}_{c}$ of the rotation group $S O(8)$ leads to an explicit non-perturbative Nicolai map [14, 15] associated with the $\mathcal{N}=8$ supersymmetry. The Nicolai map

$$
X_{0}^{i} \rightarrow W^{i}\left(X_{0}\right)=\frac{1}{2} \gamma_{\alpha \dot{\alpha}}^{i}\left[X_{0}^{\alpha}, X_{0}^{\dot{\alpha}}\right]
$$

trivializes the bosonic part of the action (11) by mapping it into a Gaussian form for the fields $W^{i}$. Moreover, the Jacobian determinant that arises from the field transformation (14) cancels exactly with the determinant that arises from integrating out the zero modes of the bilinear fermionic part of (11). The integration over the constant modes in (13) is thus formally unity. The model (1) defined with only constant field modes included is in fact the action for the low-energy dynamics of D-instantons [4 which is obtained by dimensionally reducing 10-dimensional supersymmetric Yang-Mills theory down to zero dimensions and which has been recently studied as a non-perturbative model for weaklycoupled type-IIB superstrings [19]. By T-duality, the IIA and IIB theories are equivalent, and this equivalence itself hints at a possible emergence of instanton configurations in the D-string field theory (11).

A standard diagrammatic argument (or the Schwinger-Dyson equations) shows that the leading contribution to the partition function (13) at $N=\infty$ can be evaluated as

$$
\lim _{N \rightarrow \infty} Z(R, g ; N)=\int D A \exp \left\{-\frac{N R^{2} g^{2}}{2 \pi \alpha^{\prime}} \int d \tau \oint d \sigma \operatorname{tr} F_{A, \mu \nu} F_{A}^{\mu \nu}\right\} \sqrt{\mathcal{Z}[A]}
$$

where the path integral

$$
\begin{aligned}
\mathcal{Z}[A]=\int D \phi D \phi^{\dagger} D \chi D \bar{\chi} \exp \left\{-\frac{N R^{2}}{2 \pi \alpha^{\prime}} \int d \tau \oint d \sigma \operatorname{tr}\left[\sum_{i=1}^{8}\left(\bar{\nabla}_{A} \phi_{i}^{\dagger}\right)\left(\nabla_{A} \phi^{i}\right)+2 \bar{\chi} \nabla_{A} \chi\right.\right. \\
\left.\left.-\frac{1}{g^{2}} \sum_{i<j}\left[\phi_{i}^{\dagger}, \phi^{j}\right]\left[\phi^{i}, \phi_{j}^{\dagger}\right]+\frac{2}{g} \sum_{i=1}^{8}\left(\bar{\chi}^{\alpha} \gamma_{\alpha \dot{\alpha}}^{i}\left[\phi^{i}, \chi^{\dot{\alpha}}\right]+\bar{\chi}^{\dot{\alpha}} \gamma_{\dot{\alpha} \alpha}^{i}\left[\phi_{i}^{\dagger}, \chi^{\alpha}\right]\right)\right]\right\}
\end{aligned}
$$

is defined as a functional integration over complex field configurations. We shall always ignore irrelevant (infinite) numerical factors in what follows. Here $\phi^{i}(\tau, \sigma)$ is an $N \times N$ complex matrix field in the adjoint representation of the local gauge group $U(N)$ and in the complexified vector representation $\mathbf{8}_{v} \otimes \mathbb{C}$ of the global R-symmetry group $S O(8)$. The $N \times N$ fermionic matrix $\chi(\tau, \sigma)$ is a complex, periodic 16-component fermion field in the adjoint representation of $U(N)$ whose chiral and anti-chiral components transform as Dirac-Weyl spinors in $\mathbf{8}_{s} \otimes \mathbb{C}$ and $\mathbf{8}_{c} \otimes \mathbb{C}$ under $S O(8)$. We have also introduced the chiral covariant derivatives $\nabla_{A}=\partial-i\left[A_{+}, \cdot\right]$ and $\bar{\nabla}_{A}=\bar{\partial}-i\left[A_{-}, \cdot\right]$, where $\partial=\partial_{\tau}+\partial_{\sigma}$, $\bar{\partial}=\partial_{\tau}-\partial_{\sigma}$ and $A_{ \pm}(\tau, \sigma)=A_{0}(\tau, \sigma) \pm A_{1}(\tau, \sigma)$. This doubling of the number of degrees 
of freedom in the model (an increase of the world-sheet supersymmetry from $\mathcal{N}=8$ to $\mathcal{N}=16$ ) leads, as we now show, to an explicit large- $N$ solution for the amplitude (13) as an integral over gauge orbits only.

A field $\lambda$ in the adjoint representation of the gauge group can be expanded as $\lambda=\lambda_{a} T^{a}$, where $T^{a}$ are the Hermitian generators of $U(N)$ which are normalized as $\operatorname{tr} T^{a} T^{b}=\frac{1}{2} \delta^{a b}$ and obey the commutation relations $\left[T^{a}, T^{b}\right]=i f^{a b c} T^{c}$. Doing so in (16), the integration over the complex fermion fields gives

$$
\begin{aligned}
\mathcal{Z}[A]= & \int D \phi D \phi^{\dagger} \operatorname{det}\left\|\Delta_{A}^{a b}\left[\phi, \phi^{\dagger}\right]\right\| \\
& \times \exp \left\{-\frac{N R^{2}}{2 \pi \alpha^{\prime}} \int d \tau \oint d \sigma \operatorname{tr}\left(\sum_{i=1}^{8}\left(\bar{\nabla}_{A} \phi_{i}^{\dagger}\right)\left(\nabla_{A} \phi^{i}\right)-\frac{1}{g^{2}} \sum_{i<j}\left[\phi_{i}^{\dagger}, \phi^{j}\right]\left[\phi^{i}, \phi_{j}^{\dagger}\right]\right)\right\}
\end{aligned}
$$

where $\Delta_{A}^{a b}\left[\phi, \phi^{\dagger}\right]$ is the matrix differential operator

$$
\Delta_{A}^{a b}\left[\phi, \phi^{\dagger}\right]=\left(\begin{array}{cc}
\nabla_{A}^{a b} \delta_{\alpha \beta} & -\frac{i}{g} f^{a b c} \sum_{i} \gamma_{\alpha \dot{\alpha}}^{i} \phi_{i, c}^{*} \\
-\frac{i}{g} f^{a b c} \sum_{i} \gamma_{\dot{\alpha} \alpha}^{i} \phi_{c}^{i} & \bar{\nabla}_{A}^{a b} \delta_{\dot{\alpha} \dot{\beta}}
\end{array}\right)
$$

and the ultra-local functional determinant in (17) is over both the $U(N)$ indices and the chiral-antichiral indices of $\operatorname{spin}(8)$. For this extended model with $\mathcal{N}=16$ supersymmetry, the Nicolai map is defined by the non-analytic field transformation

$$
\xi^{i}=\nabla_{A} \phi^{i}+\frac{1}{2 g} \gamma_{\alpha \dot{\alpha}}^{i}\left[\phi^{\dagger \alpha}, \phi^{\dagger \dot{\alpha}}\right] \quad, \quad \xi_{i}^{\dagger}=\bar{\nabla}_{A} \phi_{i}^{\dagger}-\frac{1}{2 g} \gamma_{\dot{\alpha} \alpha}^{i}\left[\phi^{\dot{\alpha}}, \phi^{\alpha}\right]
$$

Then the Jacobian of this transformation is $\frac{\delta\left(\xi_{a}, \xi_{a}^{*}\right)}{\delta\left(\phi_{b}, \phi_{b}^{*}\right)}=\Delta_{A}^{a b}$ and the path integral (17) becomes

$$
\mathcal{Z}[A]=\int D \xi D \xi^{\dagger} \operatorname{det}\left\|\Delta_{A}^{a b}\right\| \cdot\left|\operatorname{det}\left\|\Delta_{A}^{a b}\right\|\right|^{-1} \exp \left\{-\frac{N R^{2}}{2 \pi \alpha^{\prime}} \int d \tau \oint d \sigma \operatorname{tr} \sum_{i=1}^{8} \xi_{i}^{\dagger} \xi^{i}\right\}
$$

The trivialization of the bosonic action in (17) follows from the appropriate symmetry properties of the $\operatorname{spin}(8)$ gamma-matrices in (2) (see [13] for details).

However, the Gaussian integration in (20) is not completely trivial, because it does depend on the winding number (or degree) of the Nicolai mapping (19), i.e. on the number of distinct configurations of the original fields which are mapped into a given configuration of the free Gaussian fields with their algebraic multiplicity. Since $\xi^{i}=\xi_{i}^{\dagger}=0$ for the trivial field configuration $\phi^{i}=\phi_{i}^{\dagger}=0$, the number of times that one covers $\xi$-space as $\phi$ runs through its range can be calculated by following the zeroes of $\xi^{i}, \xi_{i}^{\dagger}$, which define the instanton and anti-instanton equations

$$
\nabla_{A} \phi_{(I)}^{i}+\frac{1}{g} \frac{\delta}{\delta \phi_{(I)}^{i}} F\left[\phi_{(I)}, \phi_{(I)}^{\dagger}\right]=0 \quad, \quad \bar{\nabla}_{A} \phi_{(I), i}^{\dagger}-\frac{1}{g} \frac{\delta}{\delta \phi_{(I), i}^{\dagger}} F^{\dagger}\left[\phi_{(I)}^{\dagger}, \phi_{(I)}\right]=0
$$

where the pre-potential is 13

$$
F\left[\phi, \phi^{\dagger}\right]=\int d \tau \oint d \sigma \sum_{i=1}^{8} \gamma_{\alpha \dot{\alpha}}^{i} \operatorname{tr} \phi^{i}\left[\phi^{\dagger \alpha}, \phi^{\dagger \dot{\alpha}}\right]
$$


Note that the instanton configurations also solve the classical equations of motion

$$
\bar{\nabla}_{A} \nabla_{A} \phi_{\mathrm{cl}}^{i}+\frac{1}{g^{2}} \sum_{i, j=1}^{8}\left(\gamma^{i} \gamma^{j}\right)_{\dot{\alpha} \dot{\beta}}\left[\phi_{\mathrm{cl}}^{\dagger \dot{\alpha}}, \phi_{\mathrm{cl}}^{\dot{\beta}}\right] \phi_{\mathrm{cl}}^{j}=0
$$

which follow from varying the bosonic action in (16) with respect to $\phi^{i}$ (and similarly for the anti-instanton configurations).

The path integral (20) calculated about $\xi^{i}=\xi_{i}^{\dagger}=0$ therefore yields an oriented sum over the different classical instanton-antiinstanton configurations,

$$
\begin{aligned}
\mathcal{Z}[A] & =\mathcal{S}_{\phi_{(I)}, \phi_{(I)}^{\dagger}} \operatorname{sgn} \operatorname{det}\left\|\Delta_{A}\left[\phi_{(I)}, \phi_{(I)}^{\dagger}\right]\right\| \\
& =\mathcal{E}_{\phi_{(I)}, \phi_{(I)}^{\dagger}} \exp \left\{\frac{\pi i}{2}\left(\zeta\left(\Delta_{A}\left[\phi_{(I)}, \phi_{(I)}^{\dagger}\right]\right)-\eta\left(\Delta_{A}\left[\phi_{(I)}, \phi_{(I)}^{\dagger}\right]\right)\right)\right\}
\end{aligned}
$$

where

$$
\begin{aligned}
& \zeta\left(\Delta_{A}\right)=\lim _{s \rightarrow 0} \frac{1}{\Gamma(s)} \int_{0}^{\infty} d t t^{s-1} \operatorname{tr}\left\|\mathrm{e}^{-t\left|\Delta_{A}\right|}\right\| \\
& \eta\left(\Delta_{A}\right)=\lim _{s \rightarrow 0} \frac{1}{\Gamma\left(\frac{s+1}{2}\right)} \int_{0}^{\infty} d t t^{(s-1) / 2} \operatorname{tr} \| \Delta_{A} \mathrm{e}^{-t \Delta_{A}^{2} \|}
\end{aligned}
$$

are, respectively, the Riemann zeta-function and Atiyah-Patodi-Singer eta-invariant which measure the spectral volume and spectral asymmetry of the self-adjoint first-order differential operator $\Delta_{A}$. The functional (24) is either a sum over discrete instanton-antiinstanton configurations or an integral over the instanton-antiinstanton moduli space $\mathcal{M}_{I}$ (the solutions to (21) modulo local gauge transformations and global R-symmetry transformations), and it is understood that zero-modes of $\Delta_{A}$ are excluded. Thus the path integral (16) represents a topological invariant of the cylindrical worldsheet embedding in $\mathbb{R}^{8}$, and, in particular, it is semi-classically exact. This property is a result of the usual nonrenormalizations in supersymmetric field theories, and it is also the generic situation in a topological field theory [15]. The $N=\infty$ vacuum amplitude of the matrix string theory can thus be written as that of an effective two-dimensional gauge theory with a local Yang-Mills kinetic term and an additional non-local term for the gauge fields,

$$
\begin{aligned}
\lim _{N \rightarrow \infty} Z(R, g ; N) \\
\quad=\sum_{\mathcal{M}_{I}} \int D A \exp \left\{-\frac{N R^{2} g^{2}}{2 \pi \alpha^{\prime}} \int d \tau \oint d \sigma \operatorname{tr} F_{A, \mu \nu} F_{A}^{\mu \nu}+\frac{\pi i}{4}\left[\zeta\left(\Delta_{A}\right)-\eta\left(\Delta_{A}\right)\right]\right\}
\end{aligned}
$$

We note that in the weakly-coupled regime of the underlying two-dimensional YangMills theory in (15) $\left(g^{2} \rightarrow \infty\right)$, the instanton equations (21) become the usual ones $\nabla_{A} \phi_{(I)}=\bar{\nabla}_{A} \phi_{(I)}^{\dagger}=0$, which are solved by a path-ordered Wilson line (so that $\phi \equiv \epsilon_{\mu \nu} F_{A}^{\mu \nu}$ ) and yield the classical field equations $\bar{\nabla}_{A} \nabla_{A} \phi_{\mathrm{cl}}=\nabla_{A} \bar{\nabla}_{A} \phi_{\mathrm{cl}}^{\dagger}=0$. The Hessian which appears in (24) is then a positive operator and so the path integral $\mathcal{Z}[A]$ just counts the total number $N_{I}=\operatorname{dim} \mathcal{M}_{I}$ of ordinary Yang-Mills instantons of the given principal 
$U(N)$-bundle over the world-sheet cylinder $\mathbb{R}^{1} \times S^{1}$. Thus the effect of the path integral (16) is to add to the Yang-Mills action in (15) a topological instanton term and we recover the world-sheet instanton configurations along with a sum over $\theta$-vacua (with $\theta \in \mathbb{Z}_{N}$ ) that we described earlier. It is intriguing that, since the ordinary Yang-Mills action in two-dimensions is a topological BF field theory in the limit of weak coupling [15, the full supersymmetric matrix model defines a topological field theory at $g^{2} \rightarrow \infty$. Thus in the weak-coupling phase the we encounter a purely topological field theory with only global degrees of freedom.

In the strong-coupling limit of the two-dimensional Yang-Mills theory $\left(g^{2} \rightarrow 0\right)$, the instanton configurations are defined by the extrema of the pre-potential (22). Then the two-dimensional matrix model is effectively independent of the gauge field $A$ and it coincides essentially with the reduced model studied in [13]. This reduced model was shown to reproduce many of the characteristic features of the matrix quantum mechanics describing the short-distance properties of D0-branes in weakly-coupled type-IIA superstring theory [2]. Again the effective theory thus obtained is essentially a topological field theory, consistent with the fact that in the free string limit the matrix model reduces to a two-dimensional supersymmetric sigma-model [8, 12, 15].

For finite $g^{2}$, the instanton equations (21) yield a one-parameter family of instanton field configurations which interpolate between ordinary Yang-Mills instantons at $g^{2} \rightarrow \infty$ (connecting the different vacua of the effective two-dimensional adjoint QCD) and the configurations which minimize the pre-potential (22) describing the characteristics of the matrix model for $\mathrm{M}$ theory in the decompactified limit $R^{2} \rightarrow \infty$ [2]. These interpolating instantons are non-perturbative configurations in the gluon sector of the theory which can form new sorts of glueball states. These states are new types of non-perturbative degrees of freedom in the full 11-dimensional $\mathrm{M}$ theory. The relevence of instanton quantum numbers has been noted in 9] for the case where the M theory matrix model is compactified on a 4torus $\left(S^{1}\right)^{4}$. There the instanton charge is identified with the wave-number of the photon field in the supersymmetric Yang-Mills theory along a new fifth direction in the quantum field theory. For a general compactification on an $n$-torus $\left(S^{1}\right)^{n}$, the type-IIA strings are $(n-1)$-dimensional domain walls wrapping around a cycle of the T-dual torus [10. In our case, in the large- $N$ limit we obtain infinitely many such solitonic walls from the $\theta$-vacua that we described before, and the corresponding world-sheet instantons are then mapped into the target space $\mathbb{R}^{8}$ by the instanton fields $\phi_{(I)}^{i}$. It is unclear though what the happens to the structure of these $\mathbb{Z}_{N}$ domains at $N \rightarrow \infty$. The instantons represent nonperturbative membrane excitations in the transverse space, and it would be interesting to see if they correspond to any previously observed $M$ theoretic membranes. In particular, it would be interesting to see if these $\theta$-vacua have anything to do with the construction of longitudinal membranes of the matrix model [0, 8]. Indeed, the physics of wrapped membranes (and other non-perturbative degrees of freedom) may be completely contained in the sum (26) over the different topological vacuum sectors of the matrix supersymmetric 
Yang-Mills theory. We note again that the strings that these configurations represent are not the usual ones obtained from the abelian sector of the theory, but are associated, in a non-trivial way via the matrix matter fields, with the topology of the full principal fiber bundle of the gauge theory.

Let us stress that the final result (26) for the vacuum amplitude is not as simple as it seems at first glance. First of all, the Nicolai map that we have exhibited is only valid at the leading order of the $1 / N$ expansion of the partition function. Thus the instanton sum (26) represents a localization of the vacuum amplitude onto the instanton moduli space associated with the large- $N$ group theory of $U(\infty)$ or $S U(\infty)$. For the full theory at finite $N$, the model is highly non-trivial. Indeed, Nicolai maps in supersymmetric gauge theories are only known in general at a perturbative level because of the highly non-local character of the mapping [14]. The only non-trivial cases where they can be constructed exactly are some low-dimensional models and topological field theories [15], in which case they localize the path integrals onto finite-dimensional objects which are topological invariants of the underlying spacetime. The large- $N$ limit that we have taken above effectively achieves this property. The second problem is that at this stage we have no idea as to the general structure of the instanton moduli space $\mathcal{M}_{I}$, or the related problem of inverting the Nicolai map to compute non-supersymmetric correlators of the matrix model at large- $N$. It is not immediate how the classification of these target space instantons will carry through in terms of the topology of the principal $U(N)$-bundle (for the world-sheet Yang-Mills instantons, they are classified by the usual first Chern cohomology classes $\left.c_{1}(U(N))=F_{A} / 2 \pi\right)$. The entire non-triviality of the theory rests in these two important aspects of the large- $N$ theory [13], and it would be interesting to deduce more precisely what the topological instantons above represent. Another important problem is using the Nicolai mapping to determine if there is a non-zero vacuum chiral condensate which may then classify the different vacuum states by ${ }_{n}\left\langle 0\left|\psi^{T} \psi\right| 0\right\rangle_{n} \sim \mathrm{e}^{2 \pi i n / N}$ [17, as they do in four-dimensional supersymmetric Yang-Mills theory. It would also be interesting to determine the properties of Wilson loop observables in this effective gauge theory, as well as other non-supersymmetric observables constructed from the dimensionally-reduced fields themselves thus making contact with objects such as D-particles and D-membranes 12 .

As we have discussed, ordinary Yang-Mills theory in two-dimensions is essentially a topological field theory with Nicolai map $\xi(A)=F_{A}^{01}$ [15], and its partition function receives contributions only from the moduli space of flat connections on the world-sheet. This reflects the relationship between topological Yang-Mills theories and conformal field theory [15]. The result (26) is an extension of this fact to the matrix string theory, and it would be interesting to see how this effective gauge theory localizes onto a finitedimensional object, given that it still represents a theory with only global degrees of freedom. It would also be interesting to connect the theory (26) with the Gross-Taylor expansion of two-dimensional Yang-Mills theory [20]. This was achieved starting from 
the heat-kernel expansion of the partition function for two-dimensional Yang-Mills theory 21

$$
Z_{\Sigma_{T}}(h, A)=\sum_{R}(\operatorname{dim} R)^{2-2 h} \mathrm{e}^{-A\left(\Sigma_{T}\right) C_{2}(R) / 2 N}
$$

in a target space $\Sigma_{T}$ which is a compact Riemann surface of genus $h$ and total area $A\left(\Sigma_{T}\right)$. Here the sum is over unitary irreducible representations $R$ of the gauge group $U(N)$ and $C_{2}(R)$ is the second Casimir of $R$. The Gross-Taylor series expands the exact solution (27) in $1 / N$ and interprets the individual terms as string maps into $\Sigma_{T}$. In this case, in order to maintain the string picture at genus $h \neq 1$ one needs to also introduce extra points on the surfaces which are weights associated with the Euler character of the Hurwitz moduli space. It would be interesting to see if the non-local additions to the Yang-Mills action in (26) corresponds in any way to the weightings by these topological invariants, thereby providing a relatively simple matrix model representation of the sum over covering maps of the Hurwitz moduli space. It would also be interesting to determine if this topological term removes the rigidity of the usual QCD strings obtained in this way, leading potentially to a statistical sum over critical string mappings. The verification of these features requires a better understanding of the instanton moduli space $\mathcal{M}_{I}$ obtained above in order to find the leading orders in $1 / N$ from (26). 


\section{References}

[1] E. Witten, Nucl. Phys. B 443 (1995) 85; J.H. Schwarz, Phys. Lett. B 367 (1996) 97.

[2] T. Banks, W. Fischler, S.H. Shenker and L. Susskind, Phys. Rev. D 55 (1997).

[3] M. Claudson and M.B. Halpern, Nucl. Phys. B 250 (1985) 689.

[4] E. Witten, Nucl. Phys. B 460 (1996) 335.

[5] U.H. Danielsson, G. Ferretti and B. Sundborg, Intern. J. Mod. Phys. A 11 (1996) 5463; D. Kabat and P. Pouliot, Phys. Rev. Lett. 77 (1996) 1004; M.R. Douglas, D. Kabat, P. Pouliot and S.H. Shenker, Nucl. Phys. B 485 (1997) 85.

[6] B. de Wit, J. Hoppe and H. Nicolai, Nucl. Phys. B 305 (1988) 545; B. de Wit, M. Lüscher and H. Nicolai, Nucl. Phys. B 320 (1989) 135; P.K. Townsend, Phys. Lett. B 373 (1996) 68.

[7] M. Berkooz and M.R. Douglas, Phys. Lett. B 395 (1997) 196; O. Aharony and M. Berkooz, Rutgers preprint RU-96-106, hep-th/9611215 (1996); G. Lifschytz and S.D. Mathur, Princeton preprint PUPT-1673, hep-th/9612087 (1996); T. Banks, N. Seiberg and S.H. Shenker, Rutgers preprint RU-96-117, hep-th/9612157 (1996); G. Lifschytz, Princeton preprint, hep-th/9612223 (1996); M.R. Douglas, H. Ooguri and S.H. Shenker, Rutgers preprint RU-97-09, hep-th/9702203 (1997).

[8] W. Taylor, Phys. Lett. B 394 (1997) 283; L. Susskind, Stanford preprint SU-ITP96-55, hep-th/9611164 (1996); O.J. Ganor, S. Ramgoolam and W. Taylor, Princeton preprint PUPT-1668, hep-th/9611202 (1996); S. Kachru and E. Silverstein, Rutgers preprint RU-96-114, hep-th/9612162 (1996); N. Kim and S.-J. Rey, Stanford preprint SLAC-PUB-7396, hep-th/9701139 (1997); S. Sethi and L. Susskind, Stanford preprint SU-ITP-97-6, hep-th/9702101(1997); D. Berenstein and R. Corrado, Texas preprint UTTG-04-97, hep-th/9702108 (1997).

[9] M. Rozali, Texas preprint UTTG-06-97, hep-th/9702136 (1997); W. Fischler, E. Halyo, A. Rajaraman and L. Susskind, Stanford preprint SLAC-PUB-7431, hepth/9703102 (1997).

[10] T. Banks and N. Seiberg, Rutgers preprint RU-97-06, hep-th/9702187 (1997); P.M. Ho and Y.-S. Wu, Utah preprint UU-HEP/97-01, hep-th/9703016 (1997); Y. Imamura, Kyoto preprint KUNS-1436, hep-th/9703077 (1997).

[11] L. Motl, Charles University preprint HEP-UK-0003, hep-th/9701025 (1997).

[12] R. Dijkgraaf, E. Verlinde and H. Verlinde, CERN preprint CERN-TH/97-34, hepth/9703030 (1997).

[13] I.I. Kogan, G.W. Semenoff and R.J. Szabo, Mod. Phys. Lett. A 12 (1997) 183.

[14] H. Nicolai, Phys. Lett. B 89 (1980) 341; 117 (1982) 408; Nucl. Phys. B 176 (1980) 419; K. Dietz and O. Lechtenfeld, Nucl. Phys. B 255 (1985) 149; O. Lechtenfeld, Nucl. Phys. B 274 (1986) 633.

[15] D. Birmingham, M. Blau, M. Rakowski and G. Thompson, Phys. Rep. 209 (1991) 129.

[16] D.J. Gross, I.R. Klebanov, A.V. Matytsin and A.V. Smilga, Nucl. Phys. B 461 (1996) 109.

[17] I.I. Kogan and A.R. Zhitnitsky, Nucl. Phys. B 465 (1996) 99. 
[18] E. Witten, Nuovo Cim. A 51 (1979) 325; L.D. Paniak, G.W. Semenoff and A.R. Zhitnitsky, Nucl. Phys. B 487 (1997) 191.

[19] N. Ishibashi, H. Kawai, Y. Kitazawa and A. Tsuchiya, KEK preprint KEK-TH503, hep-th/9612115 (1996); M. Li, Chicago preprint EFI-96-49, hep-th/9612222 (1996); I. Chepelev, Yu. Makeenko and K. Zarembo, ITEP preprint ITEP-TH07/97, hep-th/9701151 (1997); A. Fayyazuddin and D.J. Smith, Niels Bohr Institute preprint NBI-HE-97-02, hep-th/9701168 (1997); A. Fayyazuddin, Yu. Makeenko, P. Olesen, D.J. Smith and K. Zarembo, Niels Bohr Institute preprint NBI-HE-97-09, hep-th/9703038 (1997); B. Sathiapalan, Taramani preprint IMSC/97/03/10, hepth/9703133 (1997).

[20] D.J. Gross, Nucl. Phys. B 400 (1993) 161; D.J. Gross and W. Taylor, Nucl. Phys. B 400 (1993) 181; 403 (1993) 395.

[21] B. Rusakov, Mod. Phys. Lett. A 5 (1990) 693. 